\title{
Realização de atividades extraclínicas pelas equipes de saúde bucal do Brasil
}

\author{
Performance of extra clinical activities by oral health teams in Brazil
}

Anderson Falcão Tambara ${ }^{a}$

Orcid: https://orcid.org/0000-0002-8851-6647

Orlando Luiz do Amaral Junior ${ }^{b}$

Orcid: https://orcid.org/0000-0002-6611-3871

\author{
Gabriele Rissotto Menegazzo ${ }^{c}$ \\ Orcid: https://orcid.org/0000-0002-4181-0267
}

Jessye Melgarejo do Amaral Giordani ${ }^{d}$

Orcid: https://orcid.org/0000-0002-3825-9734

\begin{abstract}
Resumo
Introdução: A atividade extraclínica desenvolvida pela equipe de saúde bucal pode ser considerada toda a atividade realizada fora do âmbito da clínica odontológica, capaz de fortalecer as ações de promoção da saúde bucal na população. Objetivo: O objetivo deste estudo foi analisar quais fatores individuais e contextuais estão associados à maior realização de atividades extraclínicas pelas equipes de saúde bucal participantes do Programa Nacional de Melhoria do Acesso e da Qualidade da Atenção Básica (PMAQ-AB) no Brasil. Materiais e Métodos: Trata-se de um estudo observacional transversal realizado a partir de dados de 18.333 equipes de saúde bucal do Sistema Único de Saúde e dos municípios aos quais essas equipes de saúde estavam implantadas. $O$ desfecho utilizado foi a realização de atividades extraclínicas e as variáveis de exposição foram organizadas nas dimensões de nível contextual e nível individual do processo de trabalho. Foi utilizado regressão de Poisson multinível para avaliar a associação entre as vaiáveis. Resultados: A prevalência de realização de atividades extraclínicas pelas equipes de saúde bucal foi $54,2 \%$. Encontrou-se associação entre maior realização de atividades extraclínicas e maior porte populacional, maior Índice de Desenvolvimento Humano, maior cobertura de saúde bucal voltada à realização de atividades de cunho preventivo, em equipes que realizam atividades de educação em saúde, atividades de educação permanente têm apoio institucional e possuem um processo de longitudinalidade do cuidado. Conclusão: Este estudo reforça fatores relevantes na implementação de um modelo de assistência à saúde que aprimora os dispositivos da Clínica Ampliada.
\end{abstract}

Palavras-chave: avaliação em saúde; planejamento em saúde; saúde da família; saúde bucal.

\begin{abstract}
Introduction: The extra clinical activities undertaken by oral health teams comprise all actions developed outside dental clinic scope and which strengthens health promotion. Aim: This study aimed to verify the individual and contextual factors associated with the execution of extra clinical activities by oral health teams which are part of the National Program for Improving Access and Quality of Primary Care (PMAQ-AB), in Brazil. Materials and Methods: This is a multilevel cross-sectional observational study with data from 18,333 oral health teams of the Unified Health System as well as from the municipalities where these health teams were located. The outcome was extra clinical activities and the exposure variables were grouped in the contextual and individual levels of the working process. Poisson regression
\end{abstract}

\footnotetext{
a Programa de Pós-Graduação em Ciências Odontológicas, Universidade Federal de Santa Maria, RS, Brasil. E-mail: anderson_tambara@hotmail.com

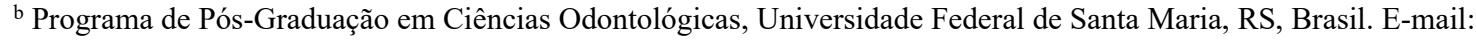
orlandodoamaraljr@gmail.com

c Programa de Pós-Graduação em Ciências Odontológicas, Universidade Federal de Santa Maria, RS, Brasil. E-mail: gabi_menegazzo@hotmail.com

d Programa de Pós-Graduação em Ciências Odontológicas, Universidade Federal de Santa Maria, RS, Brasil. E-mail: jessyesm@hotmail.com
} 
models were performed to verify the association between variables. Results: The prevalence of extra clinical actions by the oral health teams was $54.2 \%$. It was found association between greater execution of extra-clinical activities and larger population size, higher Human Development Index, greater population coverage by oral health which advocate preventive activities, in teams which perform health education activities, permanent education activities, receive institutional support and featuring a process of longitudinal care. Conclusion: This study reinforces relevant factors in the implementation of a health care model which enhance the devices of the Extended Clinic.

Keywords: family health; health evaluation; health planning; oral health.

\section{Introdução}

A avaliação da qualidade dos serviços de saúde pode ser compreendida como a análise dos componentes que fazem parte da produção desses serviços, na mensuração de aspectos de estrutura, processos e resultados, como proposto pela tríade de Donabedian ${ }^{1}$. A dimensão de estrutura engloba os recursos humanos, físicos e financeiros usados para fornecer o cuidado à saúde, assim como os parâmetros organizacionais e os meios de financiamento desses recursos. O processo abrange as ações constitutivas da atenção à saúde, incluindo a organização do processo de trabalho na oferta dos serviços de saúde. Já os resultados referem-se às mudanças nas condições de saúde da população que foram promovidas pelos cuidados recebidos ${ }^{2}$.

No Brasil, uma ação importante no planejamento de ações de avaliação e monitoramento em saúde foi desenvolvida pelo Ministério da Saúde através do Programa Nacional de Melhoria do Acesso e da Qualidade da Atenção Básica (PMAQ$\mathrm{AB})$, que objetiva fomentar a ampliação do acesso e a melhoria da qualidade da atenção em saúde com a garantia de um padrão de qualidade, permitindo maior transparência e efetividade das ações governamentais direcionadas à atenção básica. ${ }^{3}$ A Atenção Básica, por sua vez, envolve um conjunto de ações e serviços, e exerce um dos principais papéis dos sistemas de saúde, servindo como porta de entrada para serviços prestados a toda a população, com vistas à redução de problemas de saúde comuns ${ }^{4}$.

Dentre as ações avaliadas pelo PMAQ-AB, encontram-se as do processo de trabalho das equipes da atenção primária à saúde, em que, neste nível de atenção, as equipes de saúde bucal devem também desenvolver ações com os demais membros da equipe ${ }^{3}$. Dentre essas ações, a Atividade Extraclínica (AEC) desenvolvida pela equipe de saúde bucal pode ser considerada toda a atividade desenvolvida fora do âmbito da clínica odontológica e que fortaleça as ações de promoção da saúde para a população, sendo exemplo dessas, a participação em reuniões de equipe, a realização de visitas domiciliares e a educação em saúde, atividades básicas para esse processo de trabalho ${ }^{5-7}$. Essas atividades fazem parte de um modelo de atenção focado na saúde da família e fazem parte do modelo de atenção à saúde proposto pela Política Nacional de Saúde Bucal do Brasi1 ${ }^{8,9}$.

No entanto, a literatura ainda é escassa em relação às características que influenciam na organização do processo de trabalho das equipes de saúde para ofertarem AEC, e em recente avaliação, verificou-se que a condição social, demográfica e econômica dos municípios se associa com o desempenho das equipes de saúde $^{10}$. Porém, em relação à prática dessas atividades pelas equipes de saúde bucal, não se tem conhecimento de estudo que quantifique esse importante processo do sistema de saúde. Nesse contexto, o objetivo deste estudo é analisar quais fatores individuais e contextuais estão associados a uma maior adesão à realização de atividades extraclínicas pelas equipes de saúde bucal participantes do PMAQ-AB no Brasil, no ano de 2014. 


\section{Materiais e Métodos}

\section{Delineamento e amostra do estudo}

Trata-se de um estudo observacional transversal realizado a partir de dados das equipes de saúde bucal e dos municípios participantes do PMAQ-AB, no Brasil, em 2014. Nesse período o Brasil possuía 25.362 Equipes de Saúde Bucal em seu Sistema Único de Saúde ${ }^{9}$. O presente estudo avaliou 18.333 equipes, representando $77,2 \%$ das equipes de saúde bucal (ESB) brasileiras no ano de 2014. A coleta de dados foi realizada através de entrevistas estruturadas, que foram respondidas em 94,4\% dos casos pelos cirurgiões dentistas, 4,3\% pelos auxiliares de saúde bucal e 1,3\% pelos técnicos de saúde bucal membros de ESB participantes do PMAQ-AB ${ }^{3}$.

Os avaliadores foram treinados uniformemente por meio da utilização de um manual de campo elaborado pelo Departamento de Atenção Básica e pelas Instituições de Ensino e Pesquisa parceiras do PMAQ- $\mathrm{AB}^{3}$. Foram utilizados tablets, os quais continham um aplicativo com o instrumento padronizado e testado previamente. Após as coletas, os dados foram enviados via internet a um servidor do Ministério da Saúde para que fossem validados $^{3}$. Este estudo utilizou, portanto, os microdados do módulo VI PMAQ-AB referente às informações sobre processos de trabalho da equipe e sobre a organização do cuidado com o usuário, além disso, a coleta de dados contextuais (características dos municípios) foi realizada através de fontes do Ministério da Saúde.

\section{Variáveis avaliadas}

A variável dependente utilizada foi definida a partir das seguintes perguntas: " $\mathrm{A}$ Equipe de Saúde Bucal realiza atividades nas escolas/creches?" caracterizando educação em saúde e com opções de resposta sim ou não; "A Equipe de Saúde Bucal realiza visita domiciliar?" com as opções de resposta sim ou não; "A Equipe de Saúde bucal participa das reuniões da equipe de atenção básica?" com as seguintes opções de resposta como sempre, às vezes ou nunca. A partir dessas respostas foi criada uma variável que leva em consideração essas questões, visto que educação em saúde, visitas domiciliares e reuniões de equipe são atividades extraclínicas básicas a serem desenvolvidas pelas $\mathrm{ESB}^{10}$. Portanto, a variável de atividades extraclínicas foi categorizada em equipes que fazem essas atividades, incluindo na categoria sim as equipes que responderam sim para as três perguntas.

As variáveis independentes contextuais (municipais) utilizadas foram: Macrorregião (norte, nordeste, centro-oeste, sul e sudeste); Porte Populacional no ano de 2010 (até 5 mil habitantes, de 5.001 a 10 mil habitantes, de 10.001 a 50 mil habitantes, de 50.001 a 100 mil habitantes, de 100.001 a 500 mil habitantes, e mais de 500 mil habitantes); Índice de Desenvolvimento Humano (IDH) em 2010 (muito alto para aqueles municípios que possuíam score de 0,800 a 1 , alto para aqueles com 0,700 a 0,799 , médio de 0,6 a 0,699 , baixo de 0,500 0,599, e muito baixo de 0-0,499); Cobertura de saúde bucal no ano de 2011 avaliada pelo número médio mensal de equipes de saúde bucal da atenção básica, para cada 3000 pessoas, em relação à população residente total no município no ano avaliado (até 50\% de cobertura e $50 \%$ ou mais), número de exodontias realizadas pelo percentual de extrações dentárias em relação à soma de procedimentos em determinado município e ano (até $7,99 \%$ ou $8 \%$ ou mais); e número de escovações supervisionadas realizadas pela proporção média mensal de participantes na ação coletiva de escovação dental supervisionada (até $7,99 \%$ e $8 \%$ ou mais). Os pontos de corte das categorizações das variáveis Porte Populacional e IDH foram baseados no IBGE $^{11}$ e os pontos de corte das categorizações das variáveis Cobertura de Saúde Bucal, Exodontia e Escovação foram baseados nos parâmetros sugeridos pelo Ministério da Saúde. ${ }^{12}$

As variáveis independentes individuais (nível equipe de saúde) 
utilizadas foram: Educação em saúde, que compreende atividades que estimulam a prevenção de doenças, a promoção da saúde e o engajamento da população, e sua participação, em assuntos relacionados à saúde através de atividades de grupo e ações em locais estratégicos, por exemplo, foi coletada através da pergunta "A agenda da equipe de saúde bucal está organizada para ofertar atividades de educação em saúde bucal no território?" com opções de resposta sim ou não; Longitudinalidade através da pergunta "A equipe de saúde bucal garante agendamento de retorno para a continuidade do tratamento de um usuário que iniciou seu tratamento?" com opções de resposta sim ou não; Educação Permanente através do questionamento de quais profissionais da equipe de saúde bucal estão envolvidos nas ações de educação permanente, categorizada em apenas um membro da ESB (o cirurgião dentista, o auxiliar ou técnico em saúde bucal) ou a equipe de saúde bucal completa; e Apoio Institucional através da pergunta "A gestão municipal realiza apoio institucional para planejamento e organização da equipe?" com opções de resposta sim ou não.

\section{Análise estatística}

Os dados foram analisados utilizando-se o programa estatístico STATA 14.0 (Stata Corporation, College Station, TX, USA). Foram realizadas análises das frequências absolutas e relativas das variáveis estudadas, além de regressão de Poisson Multinível para obtenção das razões de prevalências (RP) não ajustadas e ajustadas com seus respectivos intervalos de confiança de $95 \%$ (IC 95\%). A modelagem foi feita de forma hierárquica em dois estágios realizada para avaliação do contexto e das variáveis individuais, em que o modelo 1 incluiu apenas as variáveis contextuais no ajuste dentro do próprio bloco contextual e o modelo 2 incluiu além das variáveis contextuais as variáveis de processo de trabalho, conforme idealizado pelo modelo hipotético criado a partir do modelo teórico de Donabedian $(1988)^{2}$ demonstrado na Figura 1.

Figura 1 - Modelo hipotético para atividades extraclínicas realizadas pelas equipes de saúde bucal da atenção básica à saúde, de acordo com adaptação de modelo teórico de Donabedian $(1988)^{2}$.

Nível Contextual

Nível Individual

(Municipal)

(Equipes de Saúde)

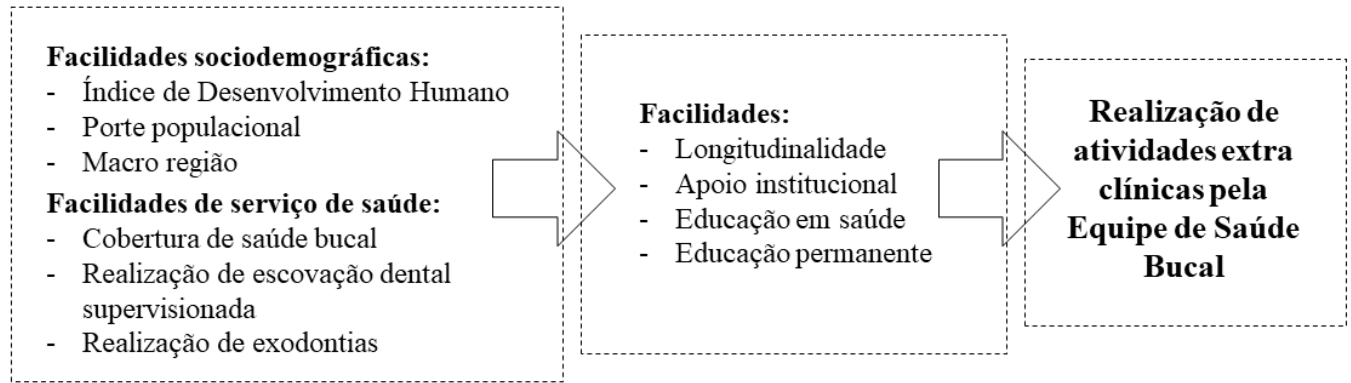

Fonte: Donabedian (1988)

\section{Preceitos éticos}

$\mathrm{O}$ protocolo de pesquisa foi submetido e aprovado pelo Comitê de Ética em Pesquisa da Universidade Federal de
Santa Maria sob número do parecer 2.887.912 e certificado de apresentação para apreciação ética número 96828618.4 .0000 .5346$. 


\section{Resultados}

No total, 19.946 ESB participaram da avaliação externa do PMAQ-AB, dessas, 1.613 foram excluídas por não apresentarem informações para atividades extraclínicas, ou seja, não possuíam dados para o desfecho investigado (taxa de resposta de 91,9\%). Portanto, para este estudo foram incluídas 18.333 equipes de saúde bucal de 4.359 municípios do Brasil. A prevalência geral de realização de atividades extraclínicas pelas equipes de saúde bucal foi 54,2\% (IC 95\%: 53,5-54,9). Quando as variáveis que compõem o desfecho foram analisadas desagregadas, os resultados mostraram que $66,2 \%$ das equipes sempre participavam das reuniões de equipe, $78,2 \%$ realizavam visitas domiciliares e $92,5 \%$ realizavam atividades na escola.
A Tabela 1 mostra a distribuição da amostra, prevalência bruta de atividades extraclínicas e razão de prevalência não ajustada e ajustada para realização de atividades extraclínicas pelas equipes de saúde bucal, segundo características contextuais. A maioria das equipes se localizava em municípios da região nordeste $(42,8 \%)$, com até 50 mil habitantes $(61 \%)$, IDH alto ou muito alto (43\%) e com cobertura de saúde bucal de mais de 50\% (67\%). A proporção média mensal entre os procedimentos realizados de escovação dental supervisionada maior ou igual a $8 \%$ era grande maioria entre as equipes (91\%), enquanto a proporção de procedimentos de exodontia em relação aos demais procedimentos maior ou igual a $8 \%$ também caracterizava a maior parte das equipes $(51,7 \%)$.

Tabela 1 - Distribuição da amostra, prevalência bruta de atividades extraclínicas e razão de prevalência não ajustada e ajustada para realização de atividades extraclínicas pelas equipes de saúde bucal segundo características contextuais, Brasil, 2014 (n = 18.333).

\begin{tabular}{|c|c|c|c|c|c|}
\hline VARIÁVEL & $\mathrm{n}(\%)$ & $\begin{array}{c}\text { Prevalência de AEC (IC } \\
95 \%)\end{array}$ & $\begin{array}{l}\text { RP não ajustada } \\
\text { (IC 95\%) }\end{array}$ & $\begin{array}{l}\text { RP ajustada } \\
\text { (IC } 95 \% \text { ) }\end{array}$ & $\mathrm{p}^{\mathrm{a}}$ \\
\hline \multicolumn{6}{|l|}{ Macrorregião } \\
\hline Norte & $1.493(7,49)$ & $44,3(41,6-47,0)$ & 1 & 1 & \\
\hline Centro-oeste & $1.721(8,63)$ & $49,0(46,6-51,5)$ & $1,07(0,94-1,21)$ & $0,95(0,84-1,08)$ & 0,43 \\
\hline Sul & $2.746(13,77)$ & $58,9(57,0-60,8)$ & $1,31(1,18-1,47)$ & $1,12(0,99-1,26)$ & 0,05 \\
\hline Sudeste & $5.431(27,23)$ & $53,2(51,8-54,5)$ & $1,17(1,05-1,29)$ & $0,98(0,89-1,10)$ & 0,84 \\
\hline Até $5 \mathrm{mil}$ & $1.281(6,42)$ & $47,4(44,6-50,2)$ & 1 & 1 & \\
\hline 5001-10mil & $2.082(10,44)$ & $42,9(40,7-45,1)$ & $0,90(0,81-1,00)$ & $0,92(0,83-1,03)$ & 0,15 \\
\hline 1001-50mil & $8.803(44,14)$ & $50,1(49,0-51,2)$ & $1,04(0,95-1,14)$ & $1,06(0,97-1,16)$ & 0,21 \\
\hline 50001-100mil & $2.399(12,03)$ & $56,1(54,0-58,1)$ & $1,16(1,04-1,28)$ & $1,19(1,07-1,33)$ & $0,00^{*}$ \\
\hline $100001-500 \mathrm{mil}$ & $3.051(15,30)$ & $63,3(61,5-65,1)$ & $1,29(1,16-1,43)$ & $1,34(1,19-1,51)$ & $0,00^{*}$ \\
\hline Mais de 500mil & $2.328(11,67)$ & $70,2(68,3-72,1)$ & $1,35(1,19-1,54)$ & $1,34(1,15-1,57)$ & $0,00^{*}$ \\
\hline Médio & $7.230(36,25)$ & $49,7(48,5-50,9)$ & $1,54(0,90-2,64)$ & $1,37(0,80-2,33)$ & 0,25 \\
\hline Alto & $7.316(36,68)$ & $58,9(57,8-60,1)$ & $1,76(1,03-3,02)$ & $1,63(0,95-2,80)$ & 0,07 \\
\hline Muito alto & $1.264(6,34)$ & $71,0(68,4-73,5)$ & $2,29(1,32-3,98)$ & $2,07(1,18-3,61)$ & $0,01 *$ \\
\hline \multicolumn{6}{|l|}{ Cobertura Saúde Bucal } \\
\hline$\leq 50 \%$ & $6.586(33,05)$ & $56,9(55,6-58,1)$ & 1 & 1 & \\
\hline$>50 \%$ & $13.343(66,95)$ & $53,0(52,1-53,8)$ & $1,01(0,96-1,07)$ & $1,22(1,14-1,30)$ & $0,00^{*}$ \\
\hline \multicolumn{6}{|l|}{ Exodontia } \\
\hline$\geq 8 \%$ & $10.302(51,66)$ & $49,5(48,5-50,5)$ & 1 & 1 & \\
\hline$<8 \%$ & $9.638(48,34)$ & $59,2(58,2-60,2)$ & $1,11(1,06-1,17)$ & $1,08(1,02-1,14)$ & $0,00^{*}$ \\
\hline \multicolumn{6}{|l|}{ Escovação } \\
\hline$<8 \%$ & $18.141(91,00)$ & $54,0(53,2-54,8)$ & 1 & 1 & \\
\hline$\geq 8 \%$ & $1.795(9,00)$ & $56,2(53,9-58,6)$ & $1,09(1,01-1,17)$ & $1,11(1,03-1,20)$ & $0,00^{*}$ \\
\hline
\end{tabular}


$\mathrm{Na}$ análise ajustada, as equipes da região nordeste apresentaram uma razão de prevalência $28 \%$ maior para práticas de atividades extraclínicas quando comparadas à região norte (RP: 1,28; IC 95\%: 1,161.41). Municípios com mais de 500 mil habitantes apresentaram uma maior razão de prevalência na realização de atividades extraclínicas quando comparados com municípios com até 5 mil habitantes (RP: 1,34; IC 95\%: 1,15-1,57). Municípios com IDH muito alto apresentaram razão de prevalência 2,07 vezes maior na realização de atividades extraclínicas (RP: 2,07; IC 95\%: 1,18-3,61) quando comparados com municípios de IDH muito baixo. E em relação ao bloco de indicadores de saúde bucal, as maiores prevalências na realização de atividades extraclínicas foram encontradas em municípios com cobertura de saúde bucal de mais de 50\% (RP: 1,22; IC 95\%: 1,14-1,30), com menos de $8 \%$ na proporção de realização de procedimentos de exodontia em relação aos demais procedimentos (RP: 1,08; IC 95\%: 1,021,14 ) e nos municípios com $8 \%$ ou mais de atividades coletivas de escovação dental supervisionada (RP: 1,11; IC 95\%: 1,03$1,20)$, em relação às suas contrapartes.

Em relação às características individuais, os resultados encontram-se na Tabela 2 onde verificamos a distribuição da amostra, prevalência bruta de atividades extraclínicas e razão de prevalência não ajustada e ajustada para realização de atividades extraclínicas pelas equipes de saúde bucal segundo características individuais. Verificou-se nessa tabela que a maioria das equipes mostrou possuir agenda organizada para ofertar ações de educação em saúde $(90,5 \%)$, garantem agendamento de retorno para a continuidade do tratamento dos usuários (91,8\%), participam das ações de educação permanente com equipe completa $(82,1 \%) \mathrm{e}$ recebem apoio istitucional da gestão municipal para planejamento e organização da equipe $(81,8 \%)$.

Tabela 2 - Distribuição da amostra, prevalência bruta de atividades extraclínicas e razão de prevalência não ajustada e ajustada para realização de atividades extraclínicas pelas equipes de saúde bucal segundo características individuais, Brasil, $2014(\mathrm{n}=18.333)$.

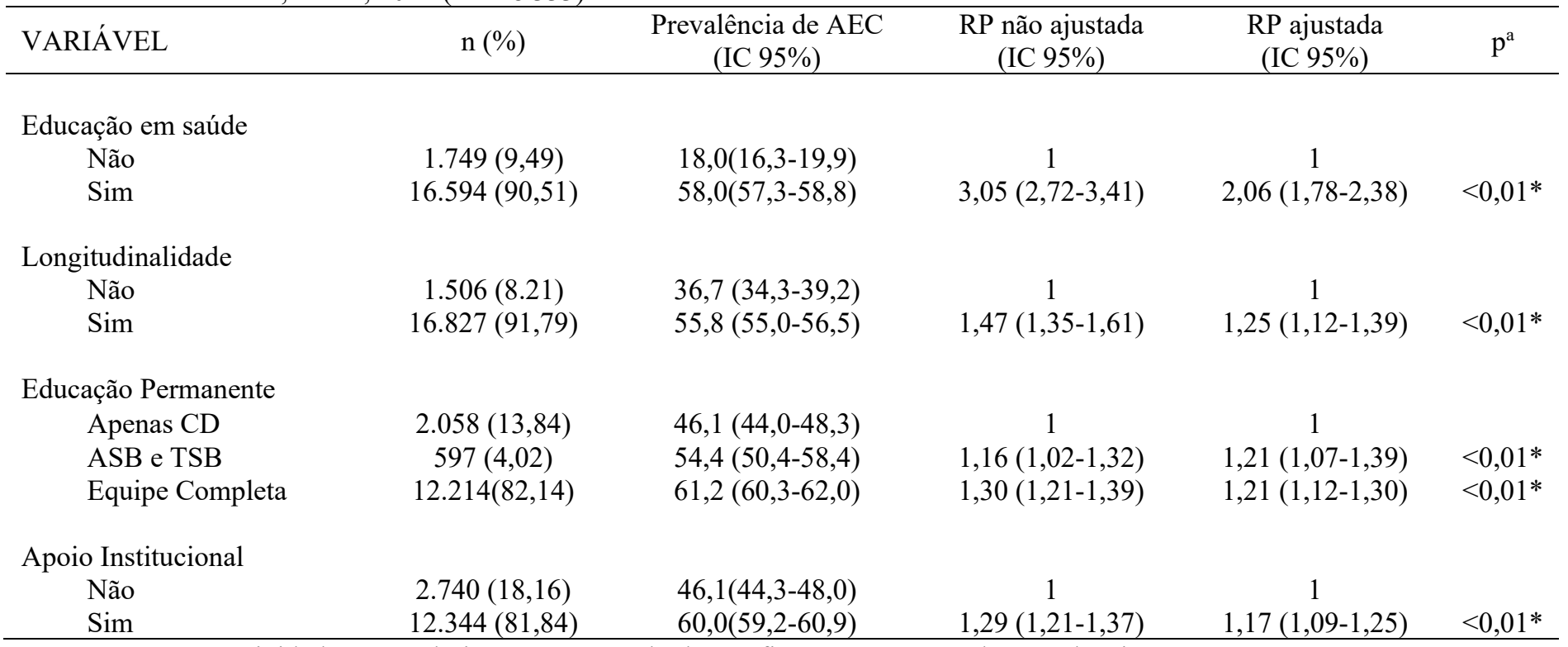

AEC: Atividades Extraclínicas; IC: Intervalo de Confiança; RP: Razão de Prevalência;

CD: Cirurgião Dentista; ASB: Auxiliar de Saúde Bucal; TSB: Técnico de Saúde Bucal.

${ }^{a}$ Valor de $\mathrm{p}$ em relação à razão de prevalência ajustada.

$* \mathrm{p}<0,05$

Fonte: Dados da pesquisa 
$\mathrm{Na}$ análise ajustada, a maior realização de atividades extraclínicas foi observada entre as equipes que possuíam agenda organizada para ofertar ações de educação em saúde (RP: 2,06; IC95\%: 1,782,38 ) em relação às equipes que não realizavam essas ações. As equipes que garantiam agendamento de retorno para a continuidade do tratamento dos usuários possuíam maior prevalência de realização de atividades extraclínicas (RP: 1,25; IC95\%: 1,12-1,39) do que as equipes que não se atentavam para esse processo de longitudinalidade. Ainda, as ESB que participavam das ações de educação permanente com equipe completa apresentaram $21 \%$ maior prevalência de realização de atividades extraclínicas (RP: 1,21 ; IC95\% 1,12-1,30) e as que recebiam apoio istitucional da gestão municipal para planejamento e organização da equipe apresentavam $17 \%$ maior prevalência de realização de atividades extraclínicas (RP: 1,17; IC95\%: 1,09-1,25), ambas em relação às suas contrapartes.

\section{Discussão}

Este estudo identificou quais características contextuais (municipais) e de processo de trabalho das equipes que estão associadas à realização de atividades extraclínicas na Atenção Básica do Brasil. Encontrou-se associação entre maior realização de atividades extraclínicas e ser uma equipe pertencente à macrorregião brasileira do nordeste, ter maior porte populacional, ter maior IDH, ter maior cobertura de saúde bucal, ter suas atividades voltadas à realização de atividades de cunho preventivo, pertencer a equipes que realizam atividades de educação em saúde com a população, que realizam atividades de educação permanente, que têm apoio institucional e que possuem um processo de longitudinalidade do cuidado. É importante que atividades extraclínicas sejam realizadas pelas ESB, visto que fazem parte do processo de trabalho da Atenção Primária à Saúde proposto pelo sistema de saúde brasileiro, levando a um modelo mais integral da atenção ${ }^{4}$.

Após análise ajustada e considerando as cinco macrorregiões brasileiras, foi possível observar que a região nordeste foi a única região significativamente associada com maior razão de prevalência para realização de atividades extraclínicas, em relação à região norte. Esse fato pode ser explicado pela região ter a maior cobertura populacional de Equipes de Saúde Bucal brasileira ${ }^{13}$. Porém, é importante ressaltar que embora a região tenha o maior número de ESB e seja a região que mais realiza atividades extraclínicas, as necessidades assistenciais são diferentes entre as regiões brasileiras e o número de equipes embora maior, pode não refletir a real melhoria do acesso da população aos serviços de saúde bucal ${ }^{14}$. Deve-se refletir também que só o aumento do número de equipes não é suficiente para melhorar o acesso da população aos serviços de saúde bucal, sendo esse um processo social que não deve ser resultante apenas de práticas odontológicas ${ }^{14}$.

Em relação ao porte populacional, os municípios menores apresentaram menor prevalência de realização de atividades extraclínicas. Os achados vão de encontro à literatura ${ }^{15}$. Esse fato pode ser explicado pelas desigualdades intermunicipais desfavoráveis aos municípios menores, o que explicita a necessidade de compreender o papel da gestão como apoiadora das $\mathrm{ESB}^{10}$. Nesse sentido, cabe refletir a respeito da importância da qualificação da gestão com apoio do Ministério da Saúde e das Secretarias Estaduais de Saúde, principalmente aos municípios menores e com baixa capacidade de resposta dos sistemas de saúde. Além disso, é importante que a regionalização seja efetiva, e que se organizem as redes de atenção em saúde de forma equânime incluindo os municípios ${ }^{16}$. Devemos considerar que municípios de menor porte apresentam uma maior rotatividade de profissionais nas equipes e cobertura de extensas áreas rurais, fatores 
esses que podem dificultar a realização de práticas extraclínicas ${ }^{17}$.

Embora tenha se observado uma tendência redistributiva da política de transferência de recursos financeiros do SUS por meio do piso de atenção básica, assim favorecendo municípios com IDH baixo $^{18}$, o presente estudo observou que a maior prevalência de atividades extraclínicas vem sendo realizadas por ESB de municípios com IDH muito alto. Esse achado vai ao encontro com resultados de estudos prévios que não encontram equidade na utilização dos serviços de saúde bucal brasileiros ${ }^{19-21}$. Nesse sentido, também é importante considerar o gradiente social como importante modulador de saúde bucal, visto que estudos, tanto em níveis individuais quanto ecológicos, vêm comprovando a relação entre desigualdades sociais e condição de saúde bucal, sugerindo a existência de iniquidades ${ }^{18,22}$.

Quanto à cobertura de saúde bucal, mais práticas de atividades extraclínicas eram realizadas pelas equipes de saúde bucal em municípios com alta cobertura. Esse achado está de acordo com os resultados encontrados em relação à saúde como um todo ${ }^{23}$ e podem ser clarificados devido à provável não sobrecarga dos profissionais, que leva à resolução mais efetiva dos problemas curativos apresentados pelos usuários do sistema. Assim, atividades extraclínicas voltadas à prevenção e foco em casos específicos podem ter uma maior ênfase no processo do cuidado.

Outros indicadores que auxiliam na avaliação dos serviços de saúde são a proporção de exodontias comparada aos demais procedimentos e a proporção de escovação dental supervisionada realizada, ambas associadas à realização de AEC pela equipe de saúde bucal, sendo maior quanto menos exodontias realizadas e mais procedimentos de cunho preventivo, ou seja, a escovação supervisionada. A análise desses indicadores é uma maneira profícua de avaliar o modelo de atenção que está sendo realizado, especialmente dentro das equipes da atenção primária à saúde, pois no Brasil se têm um modelo criado em contexto histórico centrado na assistência curativa, especializada e hospitalar, em que a mutilação é realizada de forma mais corriqueira do que os procedimentos que previnem doenças ${ }^{24}$. Portanto, é importante que, tanto as equipes quanto os usuários, sejam capazes de romper com o sistema hegemônico de atenção à saúde do país, para que as equipes sejam capazes de atuar conforme um sistema universal, capaz de valorizar a integralidade, a promoção de saúde e o cuidado humanizado, uma maneira para isso é a realização de AEC, menos procedimentos mutiladores e mais procedimentos de prevenção ${ }^{15}$.

Além dos aspectos contextuais relacionados às $\mathrm{AEC}$, é importante a observação das variáveis exploradas ao nível das equipes de saúde bucal e, sendo assim, nossos resultados apontam para maior prática de atividades extraclínicas em equipes com agenda organizada para ofertar ações de educação em saúde para os usuários, atividades essas que objetivam a apropriação do conhecimento sobre o processo saúde-doença, incluindo fatores de risco e de proteção à saúde bucal ${ }^{9}$. Essas ações de educação em saúde podem contemplar diferentes faixas etárias e locais, como por exemplo, grupos de saúde organizados dentro das unidades, grupos de terceira idade e famílias contempladas por visitas domiciliares. Nesse modelo de atividades, se propõe que o educador não apenas eduque, mas torne os sujeitos capazes de administrar sua própria saúde, lhes empoderando e dando autonomia em forma de alfabetização em saúde ${ }^{25}$. Projetos para educação em saúde são geralmente realizados de maneira multidisciplinar, necessitando planejamento e, por esses motivos, atividades extraclínicas são necessárias.

Ainda em ordem de qualidades relacionadas ao nível individual, maiores prevalências das AEC foram encontradas também entre as equipes que participavam de atividades de educação permanente com 
a presença de equipe de saúde bucal completa, ou seja, cirurgião dentista e auxiliar. A educação permanente da equipe, com a participação e integração da equipe, gestores e usuários, contribui para a transformação do processo de trabalho, com uma constante melhoria da qualidade das ações e serviços de saúde ${ }^{26}$. A equipe multiprofissional, com uma agenda que garante os momentos de educação permanente, pode proporcionar uma troca de saberes maior entre diferentes núcleos da saúde realizando ações multiprofissionais que fortalecem o serviço e contribuem para ressignificação dos processos de saúde ${ }^{27}$.

Dentre os demais fatores, maiores prevalências de realização das atividades extraclínicas foram encontradas nas equipes de saúde bucal que garantiam agendamento de retorno aos usuários. Essa ação alinha-se ao atributo da longitudinalidade da APS, que visa o acompanhamento dos usuários ao longo do tempo e a construção de vínculo e responsabilização entre profissionais e usuários $^{4,28}$. Com a possibilidade de organização da agenda de trabalho, acredita-se que se torna possível um maior controle de tempo para realização de atividades extraclínicas.

Também, as equipes que recebiam apoio institucional apresentaram maiores prevalências de realização das AEC. Essa função de apoio visa promover a análise e a gestão compartilhadas do trabalho. Nesse sentido, objetiva a democratização das instituições e procura evidenciar as relações intrínsecas entre a oferta de serviços que atendam às necessidades sociais, à configuração das organizações que os provêm e a formação subjetiva dos profissionais $^{29-31}$. Esse achado vai ao encontro de alguns estudos, que também demonstram uma mudança prática no cotidiano dos serviços, predominantemente ligada às alterações no processo de trabalho e na organização do cuidado, associadas ao apoio institucional realizado diretamente nas equipes de saúde, como a implantação e a organização do acolhimento em unidades de saúde, ou à ampliação de ações comunitárias empreendidas pelas equipes ${ }^{27}$.

Além dos achados de razões de prevalência estatisticamente significativos, a prevalência bruta de ESB que realizam atividades extraclínicas ainda se mostrou baixa. Esse fato pode ser atribuído às características inerentes à formação dos Cirurgiões Dentistas brasileiros, que acabam por valorizar o desenvolvimento de habilidades técnicas e especializadas, em detrimento das habilidades necessárias ao trabalho em equipe, o que pode refletir na falta de preparo para o trabalho em equipe e consequentemente na resistência dos profissionais para elaboração de atividades extraclínicas $^{32}$. Outra atribuição pode ser feita considerando a falta de formação de gestores, impactando consideravelmente no modelo de atenção proposto pela atenção básica aos municípios brasileiros ${ }^{33}$.

\section{Limitações do estudo}

O presente estudo possui algumas limitações. Por ser um estudo com delineamento trasversal, não estabele relações causais, apenas associações e hipóteses entre preditores e desfecho. Para este estudo tambem não foi realizada amostragem probabilística, podendo gerar viés pois a amostra é composta apenas pelas equipes de saúde que aceitaram e demonstraram desejo de participar do programa, podendo haver assim também uma superestimação dos resultados em função da tendência às respostas positivas devido à avaliação governamental realizada por meio de tais dados. É importante que possamos considerar a possibilidade de viés de informação, embora tenha ocorrido treinamento para a coleta dos dados. Também há possibilidade de confundimento, viés residual e seleção. Embora exista a possibilidade de viés de seleção, o número de equipes que não respondeu foi pequeno $(8,1 \%)$, provavelmente implicando de maneira mínima nos resultados. 
Ademais, o PMAQ não é um istrumento validado no Brasil, porém o mesmo já foi amplamente utilizado no país, sendo um instrumento de avaliação referenciado pelo próprio governo brasileiro $^{3,16}$. Como sugestão de melhoria do programa, além da ampliação de sua utilização para todas as equipes de atenção básica do Sistema Único de Saúde, a conferência dos relatos realizados pelas equipes deveria ser realizada, minimizando as possíveis discrepâncias entre os resultados e o real processo de trabalho. $\mathrm{O}$ PMAQ-AB é uma ferramenta importante na gestão dos recursos destinados à saúde, e a incorporação de tais objetivos no processo de trabalho diário das equipes torna-se importante e necessária para que todo o serviço não fique condicionado a apenas um programa $^{34}$.

\section{Conclusão}

Este foi o primeiro estudo realizado sobre as atividades extraclínicas realizadas pelas equipes de saúde bucal no Brasil, processo importante devido ao modelo de atenção focado na saúde na família, na promoção de saúde e prevenção de doenças, acabando com a necessidade de tratamento mutilador pela população ${ }^{8,10,15}$. Os resultados deste estudo demonstram que as características contextuais dos municípios e as características do processo de trabalho das equipes de saúde bucal estiveram associadas na realização de atividades extraclínicas pelas equipes participantes do PMAQ-AB no Brasil no ano de 2014. Dentre as características contextuais, destaca-se a associação da maior realização de AEC entre os municípios de maior porte populacional, maior IDH e maior cobertura de saúde bucal voltada preferencialmente à realização de atividades de cunho preventivo. Concomitante a isso, a ESB realizar atividades de educação em saúde com a população e atividades de educação permanente da equipe, ter apoio institucional e possuir um processo de longitudinalidade do cuidado também foram motivos para maior realização de AEC.

Os achados do estudo contribuem para a melhor compreensão do processo de trabalho das equipes de saúde bucal no Brasil. Além disso, reforçam fatores importantes na efetivação de um modelo de atenção alinhado às diretrizes da política nacional de saúde bucal, ou seja, que valorize os dispositivos da clínica ampliada no cuidado em saúde bucal. A avaliação realizada através do PMAQ-AB se mostra importante para o desenvolvimento de ações de saúde pública, uma vez que auxilia os gestores a organizarem a oferta dos serviços de saúde bucal assim como a qualidade do serviço ofertado, contribuindo para a integralidade dos serviços de saúde.

\section{Referências}

1. Donabedian A. The Quality of Medical Care. 1978; Science, 200(4344), 856-864.

2. Donabedian A. The Quality of Care How Can It Be Assessed? JAMA. Chicago (IL): 1988, 260: 23-30.

3. Brasil. Ministério da Saúde. Secretaria de Atenção à Saúde, Departamento de Atenção Básica. Programa Nacional de Melhoria do Acesso e da Qualidade da Atenção Básica (PMAQ-AB): manual instrutivo. Brasília; 2012.

4. Starfield B. Atenção primária: equilíbrio entre necessidades de saúde, serviços e tecnologia. Brasília: UNESCO, Ministério da Saúde; 2002.

5. Londrina. Prefeitura do Município. Autarquia Municipal de Saúde. Manual de saúde bucal; 1 ed.; Londrina, PR, 2009.

6. Farias CAP. A Política Pública como Campo Multidisciplinar. São Paulo: Unesp; Rio de Janeiro: Fiocruz; 2013, 11-21. 
7. Nickel DA, Lima FG, Silva BB. Modelos assistenciais em saúde bucal no Brasil. Cadernos de Saúde Pública; 2008, 24(2).

8. Soares CLM. Constructing public oral health policies in Brazil: issues for reflection. Brazilian oral research; 2012, 26.

9. Brasil. Ministério da saúde. Secretaria de atenção à saúde - Departamento de atenção básica- coordenação nacional de saúde bucal. Diretrizes da política nacional de saúde bucal, 2014.

10. Brasil. Ministério da saúde, Caderno da Atenção Básica - caderno 17. Brasília-DF, 2008.

11. Programa das Nações Unidas para o Desenvolvimento (PNUD). Atlas do desenvolvimento humano dos municípios. PNUD; 2013.

12. Brasil. Ministério da saúde. IDSUS, Índice de Desempenho do Sistema Único de Saúde. Disponível em: idsus.saude.gov.br/detalhadas.html; Acesso em: maio 2019.

13. Martins PHS, Amaral Júnior OL, Faustino-Silva DD, Torres LHN, Giordani JMA, Unfer B. Desigualdades na distribuição das equipes de saúde bucal no Brasil. Stomatos; 2017; 23(45): 4-13.

14. Narvai PC. Saúde bucal coletiva: caminhos da odontologia sanitária à bucalidade. Revista de Saúde Pública. 2006: p. 141-7.

15. Neves M, Giordani JM, Hugo FN. Atenção primária à saúde bucal no Brasil: processo de trabalho das equipes de saúde bucal. Ciência e saúde coletiva, 2019; 24: 1809-1820.

16. Baldai MH, Ribeiro AE, Gonçalces JR. Processo de trabalho em saúde bucal na atenção básica: desigualdades intermunicipais evidenciadas pelo PMAQ-AB. Saúde em debate; 2018; 42: 145-162.

17. Buss PM, Filho AP. A saúde e seus Determinantes sociais. Revista Saúde coletiva, Rio de Janeiro, 2007, 17(1): 77-93.

18. Fernandes JKB, Pinho JRO, Queiroz RCS, Thomaz EBAF. Evaluation of oral health indicators in Brazil: a trend towards equity in dental care? Cadernos de Saúde Pública; 2016; 32(2):1-18.

19. Gomes AMM, Thomaz EBAF, Alves MTSSB, Silva AAM, Silva RA. Fatores associados ao uso dos serviços de saúde bucal: estudo de base populacional em municípios do Maranhão, Brasil. Ciência e Saúde Coletiva; 2014; 19:629-40.

20. Baldani MH, Brito WH, Lawder JAC, Mendes YBE, Silva FFM, Antunes JLF. Individual determinants of dental care utilization among low-income adult and elderly individuals. Revista Brasileira de Epidemiologia; 2010; 13:150-62.

21. Barros AJD, Bertoldi AD. Desigualdades na utilização e no acesso a serviços odontológicos: uma avaliação em nível nacional. Ciência e Saúde Coletiva; 2002; 7:709-17.

22. Sheiham A, Alexander D, Cohen L, Marinho V, Moysés S, Petersen P, Spencer J, Watt RG, Weyant R. Global oral health inequalities: task group-implementation and delivery of oral health strategies. Advances in Dental Research; 2011; 23(2): 259-267.

23. Gomes LB, Barbosa MG, Ferla AA. Atenção Básica: olhares a partir do Programa Nacional de Melhoria do Acesso e da Qualidade (PMAQ-AB) (1st ed.). Porto Alegre: Rede UNIDA, 2016.

24. Wilkinson RG, Marmot M. Social determinants of health: the solid facts (2nd ed). Copenhagen: WHO, 2003.

25. Sentell TL, Halpin HA. Importance of adult literacy in understanding health disparities. Journal of General Internal Medicine. 2006; 21(8):862-6.

26. Pereira RCA. O trabalho multiprofissional na Estratégia Saúde da Família: estudo sobre modalidades de equipes. Rio de Janeiro, 2011. 
27. Falkenberg MB, Mendes TPL, Moraes EP, Souza EM. Educação em saúde e educação na saúde: conceitos e implicações para a saúde coletiva. Ciência e Saúde Coletiva. 2014; 19(3): 847- 52.

28. Frank BRB, et al. Avaliação da longitudinalidade em unidades de Atenção Primária à Saúde. Saúde em debate. 2015, 39(105): 400-410.

29. Campos GWS. Um método para análise e co-gestão de coletivos. a constituição do sujeito, a produção de valor de uso e a democracia em instituições: o método da roda. 2. ed. São Paulo: Hucitec, 2005.

30. Moura, RH, Luzio C A. O apoio institucional como uma das faces da função apoio no núcleo de apoio à saúde da família (nasf): para além das diretrizes. Interface, comunicação, saúde e educação, 2014; 18: 957-970.

31. Paulon SM, Pache, DF, Righi LB. Função apoio da mudança institucional à institucionalização da mudança. Interface, comunicação, saúde e educação, 2014; 18: 809-820.

32. Moretti AC, Teixeira FF, Suss FMB, Lawder JAC, Lima LSM, Bueno RE, Moysés SJ, Moysés ST. Intersetorialidade nas ações de promoção de saúde realizadas pelas equipes de saúde bucal de Curitiba-PR. Ciência e Saúde Coletiva; 2010; 15(1): 1827-1834.

33. Volschan BCG, Soares EL, Corvino M. Perfil do profissional de Saúde da Família. Revista Brasileira de Odontologia; 2002; 59(5): 314-316.

34. Medrado JRS, Casanova AO, Oliveira CCM de. Estudo avaliativo do processo de trabalho das Equipes de Atenção Básica a partir do PMAQ-AB. Saúde debate; 2015; 39(107): 1033-43.

\section{Como citar este artigo:}

Gomes THM, Garcia LAA, Casaburi LE, Santos AS. Mortalidade por suicídio da população idosa de um município do interior mineiro. Rev. Aten. Saúde. 2020; 18(64): 60-71. 